\title{
The Optometric Practice in Recessionary Times Les cabinets optométriques et la récession
}

\author{
by / par ALPHONSE CAREW
}

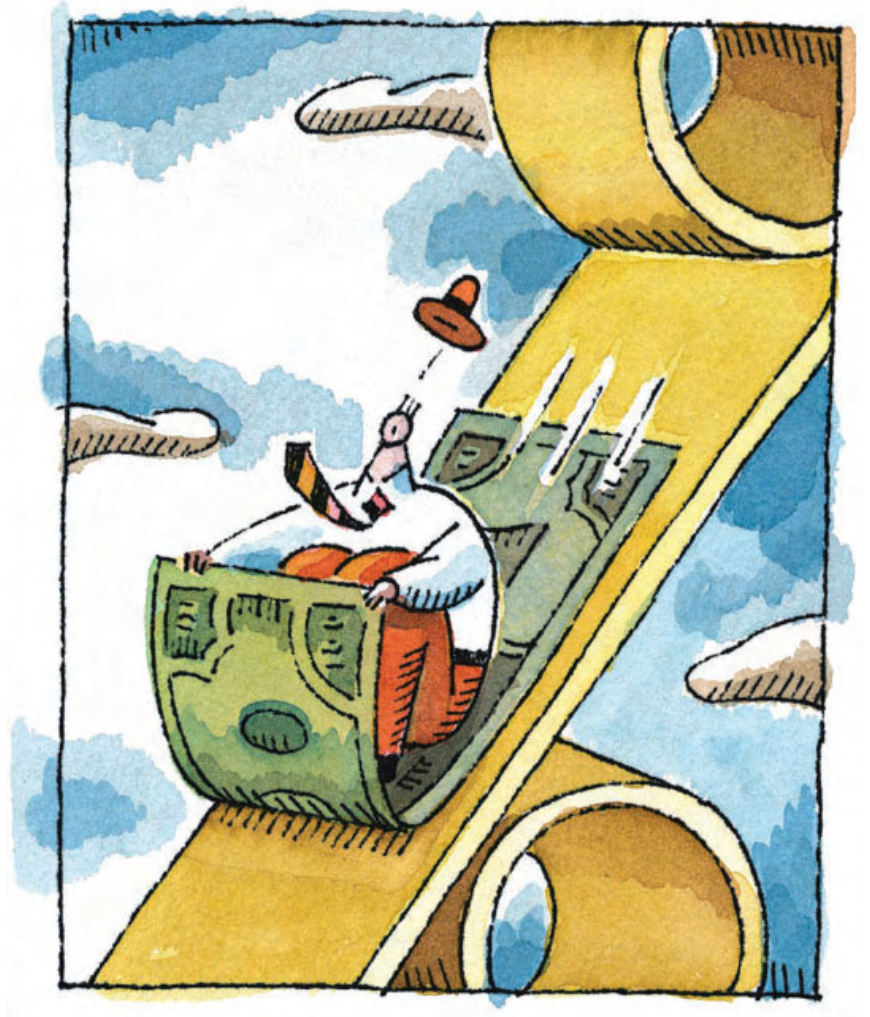

here appears to be only bad news on the economy these days with a melt-down in the stock market, a toppling in real estate values, a disaster in the banking and financial system, large increases in bankruptcies and unemployment rising to historic heights. Although the brunt of the damage is in the United States, the ripple effects are worldwide, and Canada is certainly not immune to the impact of the failing US economy, it is often said that, "when the US gets the sniffles we get the flu". In some ways however, the effects on the Canadian economy may be tempered somewhat due to the stronger regulations in our banking and insurance industries, along with the stronger social policies (unemployment, universal healthcare, third party insurance plan, etc.). But rest assured, the US is our largest trading partner and our economies are so intertwined the effects will be felt here.

What does this mean for the optometric practice? Thankfully, we enjoy a healthy profession whose demand for services continues to rise steadily. Our practices generally produce high margins and the threat of total loss or bankruptcy is quite low. However, there will be a negative impact on most practices, growth rates will weaken and possibly decline depending on the area of the country where you work. There are a few things you can do to help soften the blow of the looming financial disaster that is playing out.

In good times, and especially in bad times cash is always king. Now is a time to pay close attention to your cash flow. Make certain you are getting paid on time for services and products you provide. Keep the lion's share of your receivables under 30 days and very little over 90 days. At the same time if you can negotiate favourable terms from your suppliers allowing you to "term-out" larger purchases over 2 or 3 months this can help you free up significant cash. Make certain your inventory is at the appropriate age and size. When possible return stale or product that hasn't sold for many months, either for a refund or if that's not possible then for newer product that has a better chance of selling. Never let your inventory increase to a level higher than you absolutely need, this just needlessly ties up cash that could be put to better use.

Now would be a good time to concentrate on your core competencies. For most of us this involves providing a superior patient experience when they seek our care for their vision needs. When money is tight your patients are scrutinizing where they are spending; make certain you are providing a great value in your services 


\section{SOMETIMES WE CAN SPREAD OURSELVES TOO THIN AND OFFER EVERYTHING TO EVERYONE WHEN WE WOULD BE BETTER SERVED IF WE STUCK TO WHAT WE KNOW, AND DO, BEST.}

and products. Never loose sight of this and always support staff and policies that enhance value and consider terminating those that don't. Sometimes we can spread ourselves too thin and offer everything to everyone when we would be better served if we stuck to what we know, and do, best.

Review your expenses with an eye to cutting those that tend to creep into the practice over time but don't directly support the application of your core competencies. Cut these costs as a way to maintain profitability when your top-line or gross income comes under pressure from a slowing economy. For example, it may seem rational to have the latest magazine subscriptions or weekly office cleanings but if times start to tighten-up then some of this may have to give or at least be cut back. If that isn't possible then perhaps renegotiating contracts with your suppliers is in order. They are feeling the financial pinch as well and will work with you to keep you as a client of theirs.

Cutting staff should be the last resort. Hiring and training people to work in our practice represents a significant cost and once they are in place it is often best to try and retain them. Now, more than ever, it is important to let your staff know they are appreciated and respected, set their mind at ease if lay-offs are not part of your short-term plans. With that being said, if your salary expense is higher than it should be, and if revenue is declining, then such dramatic steps maybe necessary. Be upfront and honest with your staff on this as soon as possible, for if layoffs are necessary, your remaining staff will appreciate your honesty. On the other side of the coin, if you are in need of new staff there maybe a pool of great candidates as other practices and optical shops let people go because of their financial situation.
I'm a big proponent of getting new technology into the practice but do so very careful at this time. Any product or service that adds to the cost for your patients should be reviewed in great detail. Perhaps now is the time to hold off and be certain of where the financial trends of your practice are taking you before you commit to a large purchase. On the flip side, if you are certain that your financial outlook is very positive despite the economic news, then now might be a great time to negotiate for big ticket items.

Much has been written about marketing in slow times but most experts seem to agree that eliminating marketing is a fool's plan. Marketing can be less expensive at this time as media is cheaper to buy. Also, if your competition pulls back their advertising plans then a vacuum exists for you to fill that void and greatly increase your name recognition. As with most marketing programs it's often much cheaper and more effective to market to your existing patient base than to the general public. If you're comfortable with spending more on marketing your practice at this time then seek professional help to make sure you get the best bang for your advertising dollar.

There is strength in numbers and now might be a great time to explore the benefits of joining a buying group, for cost savings and practice management help. Practices in one location may consider joining forces in either a partnership or a cost-sharing arrangement. The healthiest and most profitable practices are multi-doctor ones taking best advantage of economies of scale.

The economy is likely to get worse before it gets better in the coming months. Stay optimistic for it will get better. Planning for the lean times now can make your practice that much healthier when rosier times return. 


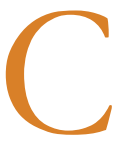

es derniers temps, nous semblons être bombardés uniquement de mauvaises nouvelles économiques : effondrement du marché boursier, basculement de l'immobilier, catastrophe dans le système bancaire et financier, nombre effarant de faillites et taux de chômage atteignant des sommets historiques. Même si les dégâts s'observent surtout aux États-Unis, l'onde de choc se fait sentir partout dans le monde et le Canada n'est certes par prémuni contre les effets d'une économie américaine vacillante. On dit souvent que lorsque les États-Unis éternuent, nous attrapons le rhume. D'une certaine façon, la réglementation plus forte à laquelle est assujetti notre secteur des banques et de l'assurance et nos politiques sociales plus vigoureuses (chômage, régime universel d'assurancemaladie, régime d'assurance de tiers, etc.) aident à tempérer quelque peu les effets sur l'économie canadienne. Mais on peut être certain que le Canada en ressentira les effets, car les États-Unis sont notre plus gros partenaire commercial.

Qu'est-ce que cela signifie pour les cabinets optométriques? Heureusement, nous avons une profession en santé, et la demande de nos services ne cesse d'augmenter. Nos cabinets procurent habituellement des marges élevées, et le risque de perte totale ou de faillite est très faible. Cependant, la récession aura un effet négatif sur la plupart des cabinets et les taux de croissance s'affaibliront et pourraient même diminuer selon la région où vous travaillez. Il y a quelques mesures que vous pouvez prendre pour atténuer le coût de la catastrophe financière qui plane à l'horizon.

En période de vaches grasses, mais surtout de vaches maigres, l'argent est toujours roi. Surveillez de prêt votre trésorerie et faites-vous payer dans les délais pour vos produits et services. Gardez la plus grande partie de vos comptes débiteurs à moins de 30 jours, et très peu à plus de 90 jours. Essayez aussi d'obtenir des modalités favorables de vos fournisseurs afin de pouvoir étaler sur deux ou trois mois vos achats importants et ainsi libérer vos liquidités. Maintenez le volume et l'âge de vos stocks à un volume et à un niveau approprié. Dans la mesure du possible, retournez les produits périmés ou ceux qui ne se vendent pas depuis longtemps soit contre remboursement, soit, si cela n'est pas possible, contre un nouveau produit qui a plus de chance de se vendre. Ne laissez jamais vos stocks augmenter plus que vous n'en avez absolument besoin puisque cela immobilise inutilement des liquidités que vous pourriez mieux utiliser ailleurs.

Pourquoi ne pas en profiter pour vous concentrer sur vos compétences de base. Pour la plupart d'entre nous, cela signifie fournir au patient une meilleure expérience lorsqu'il fait appel à nos soins. Lorsque l'argent se fait rare, vos patients scrutent davantage leurs dépenses. Pour cette raison, assurez-vous de leur fournir des produits et services d'une très grande valeur. Ne perdez jamais cela de vue : favorisez toujours les politiques et le personnel qui augmentent votre valeur et débarrassezvous de ce qui vous nuit. Parce qu'on veut trop faire, il arrive souvent qu'on se perde, alors qu'il suffirait de s'en tenir à ce qu'on connaît le mieux.

Revoyez vos dépenses en sabrant dans celles qui ont tendance à envahir votre cabinet au fil du temps sans pour autant favoriser directement vos compétences de base. Vous conserverez ainsi votre rentabilité si jamais le ralentissement de l'économie influe sur votre chiffre d'affaires ou sur votre service principal. Par exemple, il peut sembler logique de vous abonner au dernier magazine ou de faire nettoyer votre bureau chaque semaine, mais si la situation commence à se resserrer, vous devrez peut-être supprimer ou à tout le moins diminuer ce type de services. Si ce n'est pas possible, peut-être pourriez-vous renégocier vos contrats avec vos fournisseurs. Comme ils sont dans la même situation financière que vous, ils voudront vous conserver comme client.

Les mises à pied devraient être une mesure de dernier ressort. L'embauchage et la formation représentent un coût important, de sorte qu'il est souvent préférable de retenir son personnel. Plus que jamais, il est important d'exprimer votre satisfaction et votre respect à l'égard de votre personnel et de le rassurer si vous ne comptez pas procéder à des congédiements à court terme. Cela dit, si votre enveloppe salariale est plus élevée qu'elle ne devrait l'être et si vos revenus diminuent, il vous faudra peut-être recourir à de telles mesures radicales. Soyez franc et honnête avec votre personnel à ce sujet dès que possible, car si vous devez faire des mises à pied, le personnel qui restera dans votre cabinet vous 
remerciera de votre honnêteté. En revanche, si vous avez besoin de nouveaux employés, pourquoi ne pas faire appel aux excellents candidats que d'autres cabinets et magasins d'optique ont dû laisser aller à cause de la situation financière?

Même si je suis un grand partisan des nouvelles technologies, je vous incite à être très prudent en ce moment. Tout produit ou service qui augmente le coût pour vos patients devrait être examiné soigneusement. Peut-être vaut-il mieux attendre et confirmer d'abord l'orientation financière de votre cabinet avant de vous engager dans des achats importants. À l'inverse, si vos perspectives financières sont très positives malgré la conjoncture économique, peut-être est-il maintenant temps de négocier l'achat de ces technologies coûteuses.

Il s'est écrit beaucoup de choses sur le marketing lorsque l'économie ralentit, mais la plupart des experts semblent d'accord pour dire qu'il serait idiot d'éliminer toute forme de publicité. Il pourrait vous en coûter moins cher en ce moment puisque les supports médiatiques ont diminué de prix. De plus, si vos concurrents retirent leurs annonces, il y a alors un vide à combler qui pourrait mousser énormément votre image. Comme pour la plupart des programmes de marketing, il est souvent moins cher et plus efficace de cibler vos patients actuels que le public en général. Si vous ne craignez pas de dépenser davantage pour faire connaitre votre cabinet en ce moment, pourquoi ne pas demander l'aide d'un spécialiste qui vous en donnera pour votre argent.

Comme l'union fait la force, il serait peut-être grand temps d'examiner les avantages d'adhérer à un groupe d'achat pour économiser et obtenir des conseils pour la gestion de votre cabinet. Par exemple, les cabinets d'une région pourraient joindre leurs forces dans un partenariat ou conclure une entente de partage des coûts. Les cabinets les plus dynamiques et les plus rentables regroupent plusieurs professionnels qui profitent au maximum des économies d'échelle.

Il est sans doute vrai de dire que l'économie ira en empirant avant de prendre du mieux dans les mois à venir, mais restez optimiste, car la situation s'améliorera. Prévoir maintenant une période de vaches maigres peut se traduire plus tard par un avantage lorsque le beau temps reviendra.

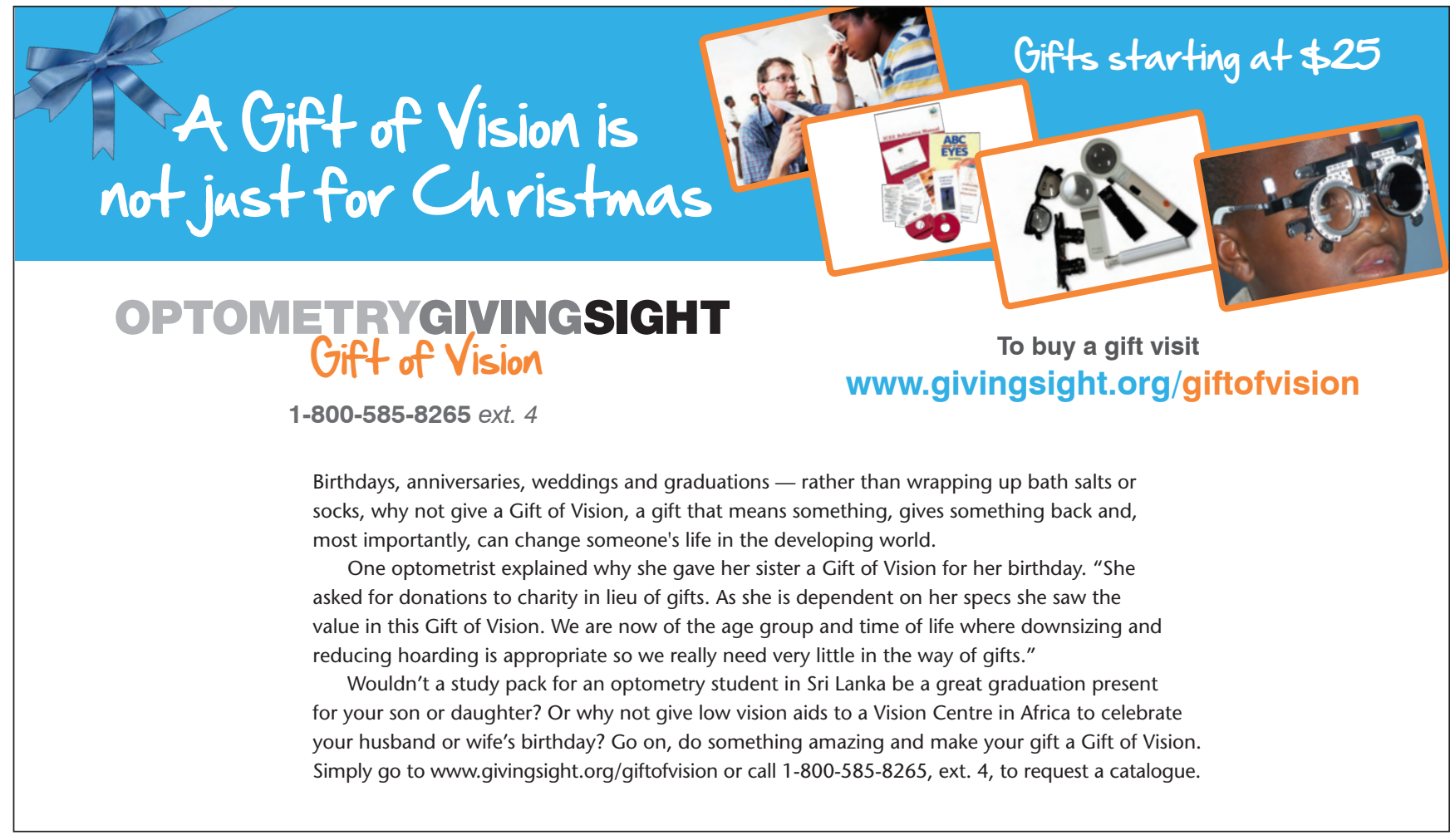

